\title{
Efficient Phase-Matched Third Harmonic Light Generation in Hexafluoroisopropanol Solutions of a Pyrimidonecarbocyanine Dye
}

\author{
W. Leupacher and A. Penzkofer \\ Naturwissenschaftliche Fakultät 11 - Physik, Universität, \\ D-8400 Regensburg, Fed. Rep. Germany \\ B. Runde and K. H. Drexhage \\ Physikalisch-Chemisches Institut, Universität, \\ D-5900 Siegen, Fed. Rep. Germany
}

Received 19 February 1987/Accepted 30 April 1987

\begin{abstract}
The phase-matched collinear third harmonic generation of picosecond laser pulses in a 0.0825 molar hexafluoroisopropanol solution of a pyrimidonecarbocyanine dye is studied. The fundamental pulses are generated in a passively mode-locked Nd-phosphate glass laser. The saturation of third harmonic generation at high intensities is investigated. The influences of two-photon absorption, excited-state absorption, and amplified spontaneous emission are discussed. For input peak intensities above $10^{11} \mathrm{~W} / \mathrm{cm}^{2}$ a third harmonic energy conversion of about $2 \times 10^{-4}$ is achieved.
\end{abstract}

PACS: $42.65 \mathrm{C}, 42.55 \mathrm{M}, 78.20$

\section{Introduction}

The anomalous refractive index dispersion of dye solutions above the $S_{0}-S_{1}$ absorption band allows the phase-matched generation of third harmonic light [1 -7] by third-order nonlinear light-matter interaction. Energy conversion efficiencies up to $\eta \simeq 2 \times 10^{-9}$ have been achieved for nanosecond pulses of a Nd-YAG-laser (peak intensity $I_{\mathrm{OL}} \simeq 10^{7} \mathrm{~W} / \mathrm{cm}^{2}$ ) in hexamethylindocarbocyanine iodide dissolved in hexafluoroisopropanol [5]. Using picosecond pump pulses of a modelocked Nd-glass laser an energy conversion of $\eta \simeq 4 \times 10^{-8}$ was obtained in 0.38 molar methylenc blue (solvent methanol) at a peak pulse intensity of $I_{\mathrm{Ot}}=3 \times 10^{9} \mathrm{~W} / \mathrm{cm}^{2}$ [7].

In order to increase the conversion efficiency, phasematchable dye solutions of low absorption coefficient, $\alpha_{3}$, at the third harmonic frequency, $v_{3}$,

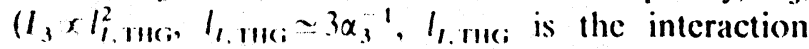
length of third harmonic generation) and high pump pulse intensities $\left(I_{3} \times l_{1}^{3}\right)$ are needed. In the present paper picosecond third harmonic generation in the basic pyrimidonecarbocyanine dye $1,3,1^{\prime}, 3^{\prime}$ tetramethyl-2,2'-dioxopyrimido-6,6'-carbocyanine hydrogen sulfate (PYC, structural formula in Fig. 2) dissolved in hexafluoroisopropanol is studied. This dye has a low absorption cross-section at the third harmonic frequency $\left(\sigma_{3} \simeq 3.5 \times 10^{-18} \mathrm{~cm}^{2}\right)$ and its third order non-linear susceptibility, $\chi_{x x x x x}^{(3)}\left(-\omega_{3} ; \omega_{1}\right.$, $\left.\omega_{\mathrm{L}}, \omega_{\mathrm{L}}\right)$, is two-photon enhanced by the strong $S_{0}-S_{1}$ absorption band around the second harmonic frequency $2 v_{\mathrm{L}}\left(v_{\mathrm{L}}=\omega_{1} / 2 \pi\right)$.

In the experiments the dependence of third harmonic generation on pump intensity and dye cell length is studied. The third-order nonlinear susceptibility, $\chi_{x x x x}^{(3)}\left(-\omega_{3} ;(1)_{1},(1)_{1},()_{1}\right)$, of the phase-matched dye solution is determined. The saturation of third harmonic generation at high pump pulse intensities is investigated. The influences of two-photon absorption, of excited-state absorption of third harmonic light, and of amplified spontaneous emission are discussed. 


\section{Experimental}

Picosecond light pulses of a mode-locked $\mathrm{Nd}$ phosphate glass laser (wavelength $\lambda_{1}=1.054 \mu \mathrm{m}$, duration $A t_{\mathrm{L}} \simeq 5 \mathrm{ps}$ ) are used for the frequency tripling experiments. The experimental set-up is depicted in Fig. 1. Amplified single picosecond light pulses are focused (lens $L_{1}$ ) to the dye sample $S$. The energy $W_{3}$ of

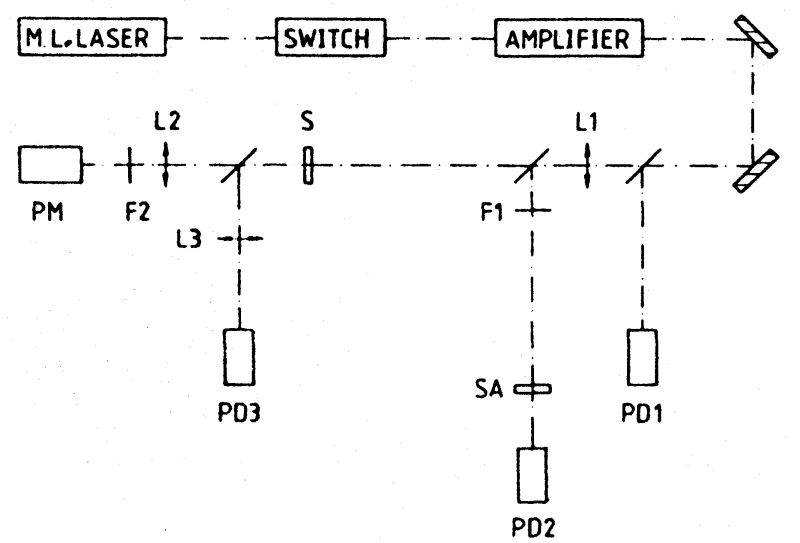

Fig. 1. Experimental arrangement. PD1-PD3, photodetectors. $P M$, photomultiplier. $L 1-L 3$, lenses, $F 1, F 2$, filters. $S A$ saturable absorber for intensity detection. $S$, third-harmonic generation sample

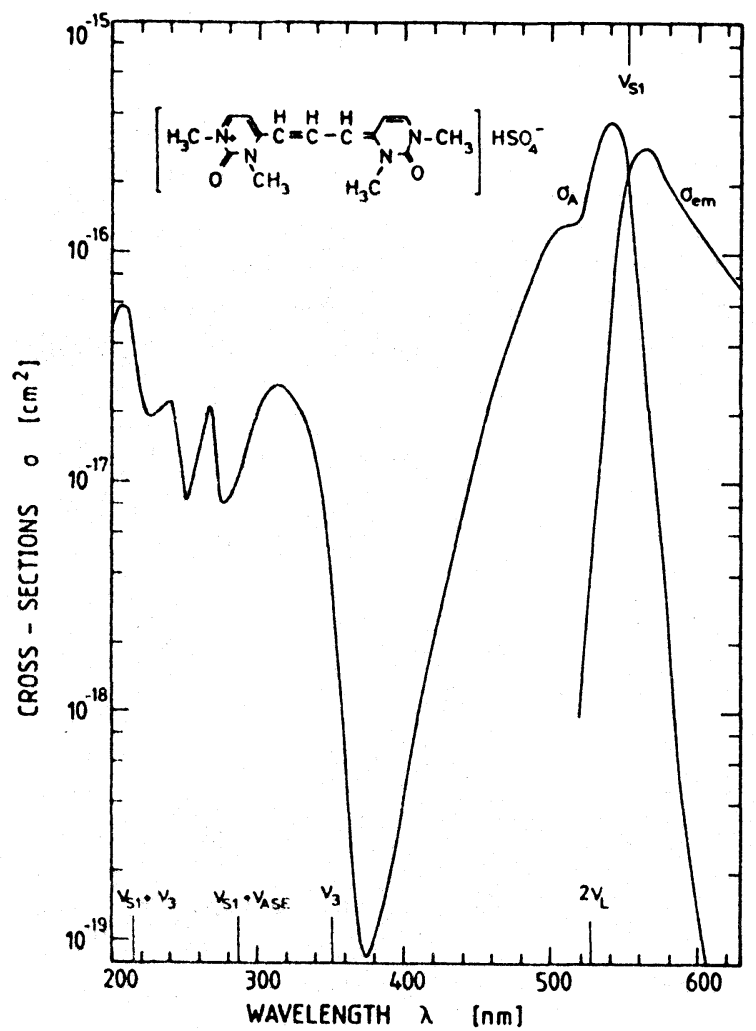

Fig. 2. Absorption and emission cross-section spectrum of 0.0825 molar PYC in 1,1,1,3,3,3-hexafluoro-2-propanol (hexafluoroisopropanol). Structural formula of the dye is inserted. Relevant wavelength positions are indicated the generated third harmonic signal is detected by photomultiplier PM. The input fundamental pulse energy $W_{\mathrm{L}}$ is registered by photodetector PD1. The peak intensity $I_{O}$. of the input pulse is detected by energy transmission measurement through a saturable absorber, $S A$, (Kodak dye No. 9860 in 1,2-dichloroethane small signal transmission $T_{0}=0.173[8]$ ) with photodetectors PD1 and PD2. The two-photon absorption of the dye in sample $S$ is monitored by energy transmission measurement with detectors PD3 and PD1. For the determination of the two-photon absorption cross-section a separate measurement was carried out with a 0.1 molar dye solution in a $1 \mathrm{~cm}$ long cell.

\section{Results}

The dye PYC (structural formula, see Fig. 2) has been a gift of Dr. U. Mayer (BASF, Ludwigshafen). The phase-matching concentration of PYC in hexafluoroisopropanol is $C_{\mathrm{PM}}=0.0825 \mathrm{~mol} / \mathrm{dm}^{3}$ (see below and Fig. 3). For this dye concentration the absorption and stimulated emission cross-section spectra are shown in Fig. 2. The absorption spectrum was measured with a conventional spectro-photometer in the wavelength region where $\sigma_{\mathrm{A}}<5 \times 10^{-17} \mathrm{~cm}^{2}$. The high $S_{0}-S_{1}$ absorption peak around $540 \mathrm{~nm}$ was determined by reflectivity measurements of parallel pola-

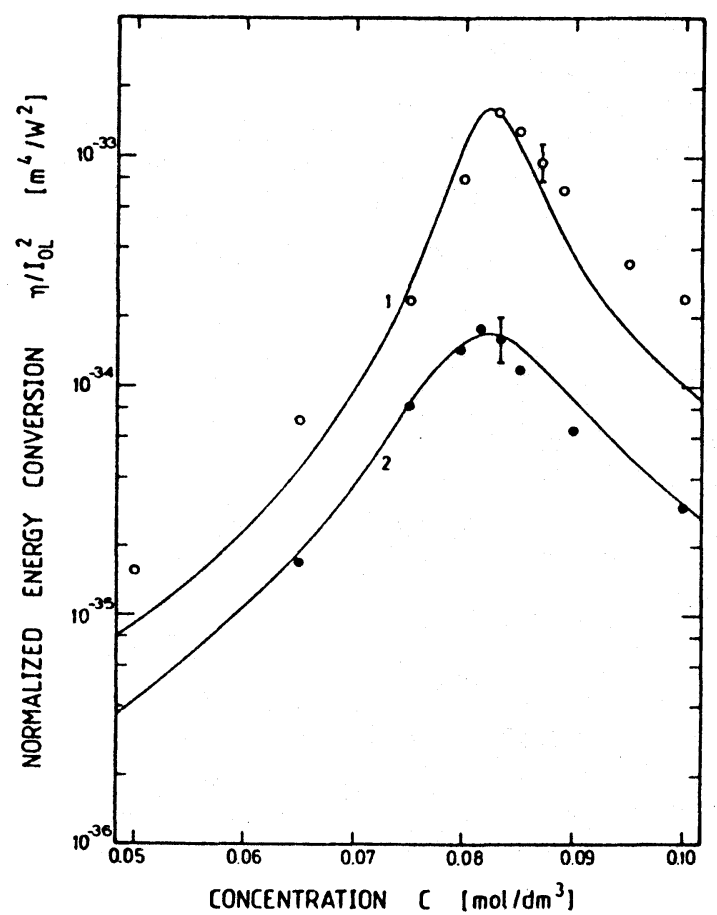

Fig. 3. Normalized third harmonic energy conversion, $\eta / I_{\text {ol. }}^{2}$, versus dye concentration. Circles, $I_{\text {ot }}=4 \times 10^{4} \mathrm{~W} / \mathrm{cm}^{2}$; dots, $I_{\mathrm{O}}=10^{11} \mathrm{~W} / \mathrm{cm}^{2}$. Curves calculated by use of $(2,3,10$, and 17) with $\left|\chi^{(3)}\right|=\left[\left|\chi_{1}^{(3)}\right|^{2}+\chi_{s}^{(3) 2}\right]^{1 / 2}[7]$. Sample length $\mid=1 \mathrm{~mm}$ 
rized light at the Brewster angle [9]. The stimulated emission cross-section spectrum, $\sigma_{\mathrm{em}}\left(C_{\mathrm{PM}}\right)$, is derived from fluorescence quantum distribution measurements $[10] . \sigma_{\mathrm{cm}}\left(C_{\mathrm{PM}}\right)$ is related to the monomer $\left(\sigma_{\mathrm{cm}, \mathrm{M}}\right)$ and closely-spaced pair $\left(\sigma_{\mathrm{em} . \mathrm{p}}\right)$ emission cross-sections by

$$
\left.\sigma_{\mathrm{em}}\left(C_{\mathrm{PM}}\right)=x_{\mathrm{M}} \sigma_{\mathrm{em}, \mathrm{M}}+x_{\mathrm{D}} \sigma_{\mathrm{em}, \mathrm{D}}\right)
$$

$x_{M}$ is the monomer mole fraction and $x_{D}$ is the mole fraction of molecules in closely-spaced pairs. The cross-sections $\sigma_{\mathrm{em}, \mathrm{M}}$ and $\sigma_{\mathrm{em}, \mathrm{D}}$ as well as the mole fractions $x_{M}=1-x_{D}$ and $x_{D}$ have been determined previously $[10]\left[x_{\mathrm{D}}\left(C_{\mathrm{PM}}\right)=0.775\right] . \sigma_{\mathrm{em}}\left(C_{\mathrm{PM}}\right)$ of $(1)$ is responsible for amplification of spontancous emission (see below).

The frequencies $2 v_{\mathrm{L}}$ and $v_{3}=3 v_{\mathrm{L}}$ are indicated in Fig. 2. The strong $S_{0}-S_{1}$ absorption band around $2 v_{L}$ is responsible for the anomalous dispersion at $v_{3}$ which

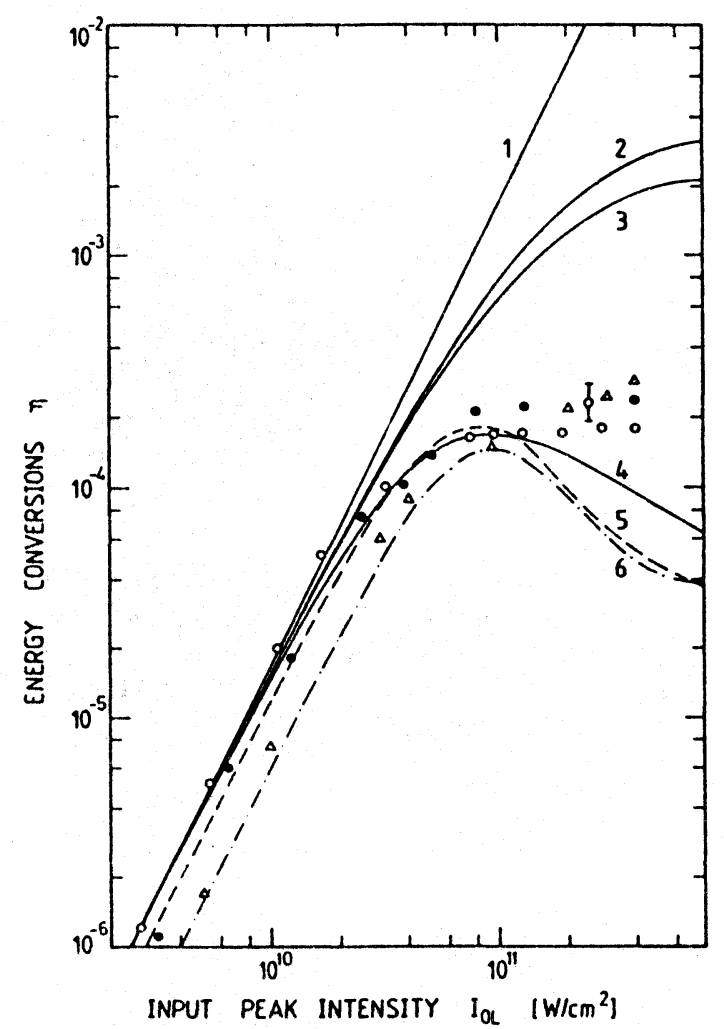

Fig. 4. Third harmonic energy conversion, $\eta$, versus input pulse peak intensity, $I_{O}$. Phase-matching dye concentration is used. Points are measured, curves are calculated. Sample lengths are $l=1 \mathrm{~mm}$ for circles $(0)$ and solid curves, $l=0.2 \mathrm{~mm}$ for dots $(\bullet)$ and dashed curve, and $l=0.1 \mathrm{~mm}$ for triangles $(\Delta)$ and dash-dotted curve. Curve 1, without effects of two-photon athsorption (Fq. 2). Curve 2, includes two-photon absorption of pumpl laser, hut without excited-state absorption (Eqs. 2 and 10). Curve 3, comprises two-photon absorption of pump laser and third harmonic light ( 2 and 13). Curves 4 6, include two-photon ahsorption and excited-state absorption without effects of amplilied spontancous cmission $[2,11$, and 17) is necessary for concentration dependent phasematching. It also causes two-photon resonant enhancement of third harmonic generation and leads to two-photon absorption. The absorption cross-section at $v_{3}$ is $\sigma_{3} \simeq 3.5 \times 10^{-18} \mathrm{~cm}^{2}$. Unfortunately the absorption minimum is located at $375 \mathrm{~nm}$ $\left[\sigma(375 \mathrm{~nm}) \simeq 8.5 \times 10^{-20} \mathrm{~cm}^{2}\right]$ and does not coincide with the third harmonic wavelength at $351.3 \mathrm{~nm}$.

The phase-matching dye concentration is found by measuring the efficiency of third harmonic generation versus concentration. In Fig. 3 the normalized energy conversion, $\eta / I_{\mathrm{OL}}^{2}=W_{3} /\left(W_{\mathrm{L}} I_{\mathrm{OL}}^{2}\right)$, versus concentration is depicted for the pump pulse intensities $I_{\mathrm{OL}} \simeq 4 \times 10^{9} \mathrm{~W} / \mathrm{cm}^{2}$ (circles) and $I_{\mathrm{OL}} \simeq 10^{11} \mathrm{~W} / \mathrm{cm}^{2}$ (dots). For both pump pulse intensities the phasematching concentration (concentration of peak normalized energy conversion) is found to be the same. The obtained value of the phase-matching concentration is $C_{\mathrm{PM}}=(0.0825 \pm 0.002) \mathrm{mol} / \mathrm{dm}^{3}$. The highintensity saturation is manifested by the reduced normalized energy conversion at the higher pump pulse intensity.

The dependence of the energy conversion, $\eta=W_{3} / W_{L}$, on the pump pulse peak intensity, $I_{O L}$, is depicted in Fig. 4 for three different sample lengths of $l=1 \mathrm{~mm}$ (circles), $0.2 \mathrm{~mm}$ (dots), and $0.1 \mathrm{~mm}$ (triangles). At low intensities the energy conversion is highest for the longest sample. At high intensities saturation (deviation from quadratic rise of energy conversion with input intensity) occurs for all three sample lengths. An energy conversion around

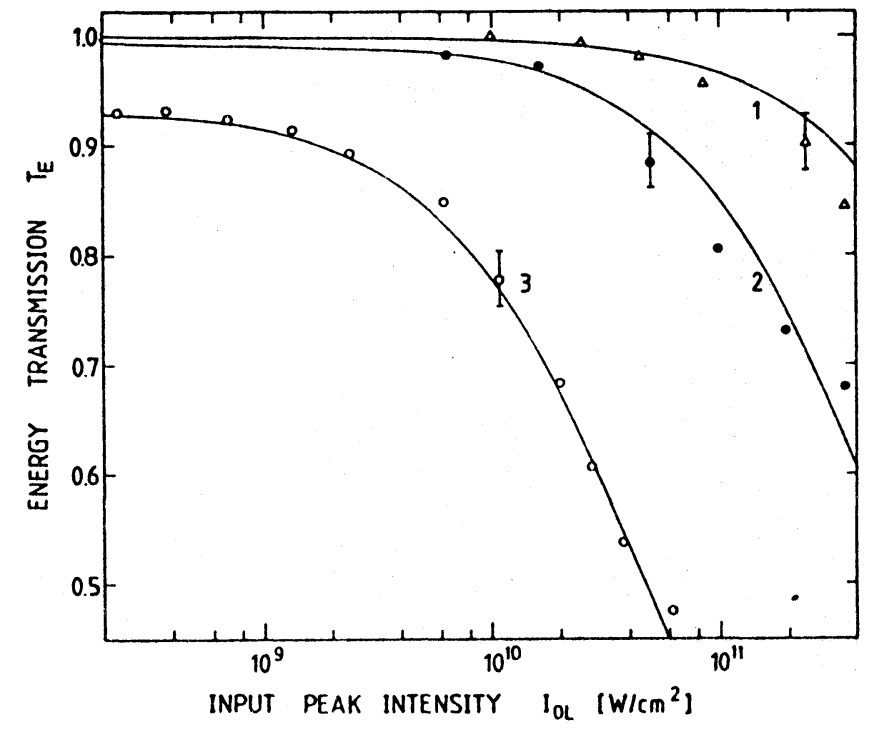

Fig. 5. Two-photon transmission through PYC in hexaflluoroisopropanol. Triangles and curve 1 : concentration $C=C_{\mathrm{PM}}$ $=0.0825 \mathrm{~mol} / \mathrm{dm}^{3}$ and sample length $l=0.2 \mathrm{~mm}$. Dots and curve 2: $C=C_{\mathrm{PM}}$ and $l=1 \mathrm{~mm}$. Circles and curve 3 : $C=0.1 \mathrm{~mol} / \mathrm{dm}^{3}$ and $l=1 \mathrm{~cm}$. Curves are calculated for $\sigma_{l .1}^{(2)}=1.8 \times 10^{44} \mathrm{~cm}^{4} \mathrm{~s}$ 
$2 \times 10^{-4}$ is achicved above pump pulse intensities of $I_{\mathrm{Ol}} \simeq 10^{11} \mathrm{~W} / \mathrm{cm}^{2}$ for all three sample lengths.

The saturation of third harmonic generation is thought to be induced by the simultaneously occurring two-photon absorption which causes pump intensity reduction and $S_{1}$-state population. The $S_{1}$-state population leads to excited-state absorption of third-harmonic light and amplified spontaneous emission (see discussion below). The results of the twophoton absorption measurements are shown in Fig. 5. The triangles belong to the phase-matching concentration and cell length of $0.2 \mathrm{~mm}$. The dots are measured for the phase-matching situation on a $1 \mathrm{~mm}$ long cell. The circles have been obtained for a 0.1 molar dye solution in a $1 \mathrm{~cm}$ long cell.

\section{Discussion}

The third-harmonic generation and the effects of twophoton absorption are discussed by use of the level model of Fig. 6. Part (a) illustrates the process of thirdharmonic generation. Part (b) depicts the two-photon absorption and the two-photon induced excited-state absorption and amplified spontaneous emission. Part (c) is responsible for two-photon absorption of the

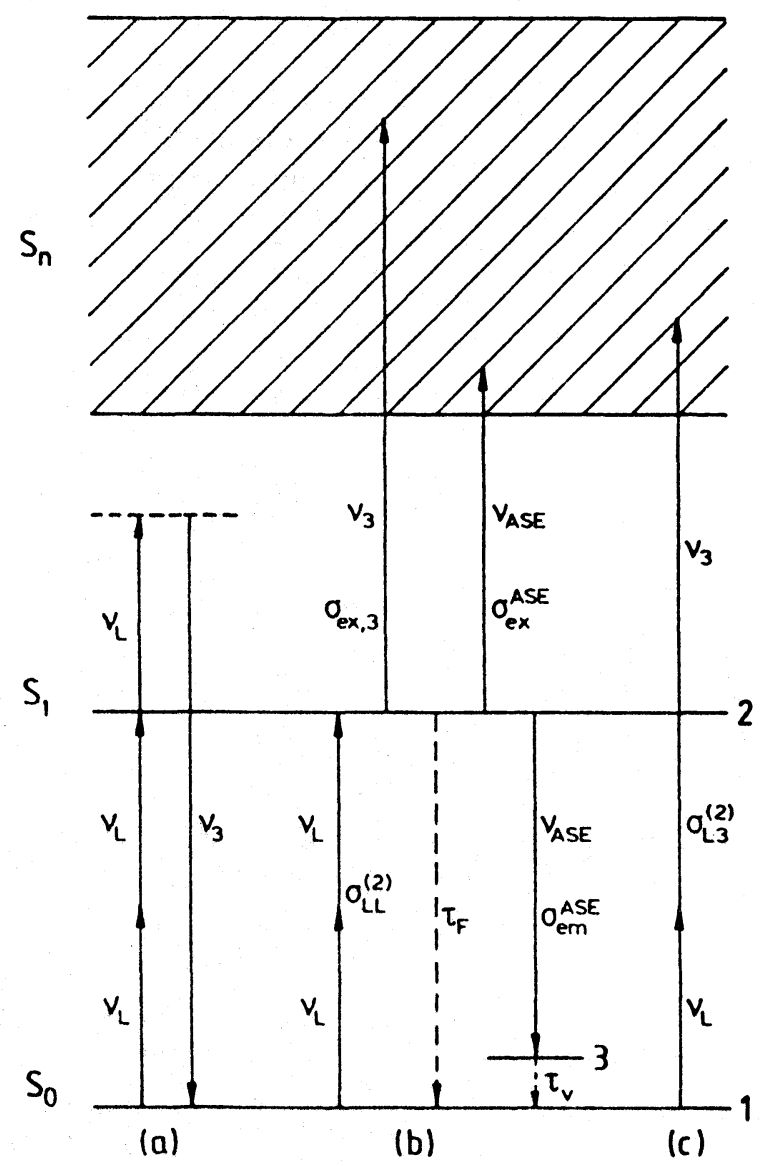

Fig. 6. Inergy level diagram

Solute: generated third harmonic light. The two-photon absorption at frequency $v_{l}$. reduces the pump pulse intensity by molecule excitation from level 1 to level 2 $\left(S_{0}-S_{1}\right.$ transition). The generated third harmonic light suffers excited state absorption by $S_{1}-S_{n}$ transition and two-photon absorption (a photon at frequency $v_{3}$ and a photon at frequency $v_{\mathrm{L}}$ are simultaneously absorbed). The $S_{1}$-state population returns to the ground-state by radiationless decay, radiative decay (spontaneous emission), and amplified spontaneous emission. The amplified spontaneous emission increases exponentially with $S_{1}$-state population

$\left\{I_{\mathrm{ASE}} \propto \exp \left[\sigma_{\mathrm{em}}^{\mathrm{ASF}}\left(N_{2}-N_{3}\right) l\right]\right\}$.

In case of fast depopulation of level $3\left(\tau_{r} \rightarrow 0\right)$ it sets an upper limit of the $S_{1}$ state population $N_{2}$ [11-14]. Excited-state absorption of the amplified spontaneous emission signal reduces the amplification of nuorescence light

$\left[I_{\mathrm{ASE}} \propto \exp \left(-\sigma_{\mathrm{ex}}^{\mathrm{ASE}} N_{2} l\right)\right]$.

The population of the $S_{1}$ state may influence the refractive index of the dye solution. The experimental points of Fig. 3 indicate that the phase-matching concentration is not measurably influenced by the pump pulse intensity. Therefore, refractive index changes are not analysed further $[15,16]$.

The experimental points of Fig. 4 indicate that up to $I_{\mathrm{OL}} \simeq 2 \times 10^{10} \mathrm{~W} / \mathrm{cm}^{2}$ the third harmonic generation is practically not influenced by two-photon absorption. For this low-intensity region the relation between energy conversion, $\eta=W_{3} / W_{\mathrm{L}}$, and third order non-

Table 1. Parameters of PYC in 1,1,1,3,3,3-hexafluoro-2-propanol. (Wavelengths $\lambda_{1}=1.054 \mu \mathrm{m}, \lambda_{3}=351.3 \mathrm{~nm}$, phase-matching concentration $C_{\mathrm{PM}}=0.0825 \mathrm{~mol} / \mathrm{dm}^{3}$ ). Transformation factors between SI and csu units are $\gamma^{(3)}(\mathrm{esu})=8.0888 \times 10^{24} \gamma^{(3)}(\mathrm{SI})$ and $\chi^{(3)}(\mathrm{esu})=\left(9 \times 10^{8} / 4 \pi\right) \chi^{(3)}(\mathrm{SI})[26]$

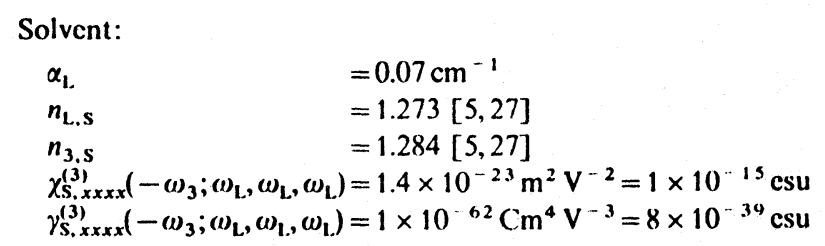

Phase-matched solution:

$$
\begin{aligned}
& \alpha_{3} \quad=177 \mathrm{~cm}^{-1}\left(\text { for } N_{2}=0\right) \\
& l_{\text {1. THG }}=3 \alpha_{3}^{-1} \quad=0.17 \mathrm{~mm} \\
& n_{\mathrm{L}}=n_{3} \quad=1.296[5,27] \\
& \mid \chi_{1}^{(3)},_{x x x x}\left(-()_{3} ;()_{1},\left(\omega_{1}, \omega_{1}\right) \mid=2.0 \times 10^{-22} \mathrm{~m}^{2} \mathrm{~V}^{-2}=1.4 \times 10^{-14} \mathrm{csu}\right.
\end{aligned}
$$

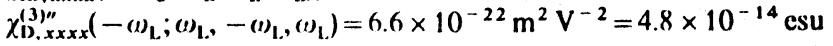

$$
\begin{aligned}
& \sigma_{3} \quad=3.55 \times 10^{18} \mathrm{~cm}^{2} \\
& \sigma_{3,2 \times} \quad=2.6 \times 10^{16} \mathrm{~cm}^{2} \\
& \sigma_{1.1}^{(2)} \quad-1.8 \times 10^{-49} \mathrm{~cm}^{4} \mathrm{~s} \\
& \left.\left.\mid \gamma_{1}^{\prime}\right)_{1}^{\prime \prime}, \ldots(-1)_{3} ;(1)_{1},(1)_{1},(1)_{1}\right)=1.7 \times 10^{59}\left(\mathrm{~cm}^{4} \mathrm{~V}{ }^{3}=1.4 \times 10^{34} \mathrm{esu}\right.
\end{aligned}
$$

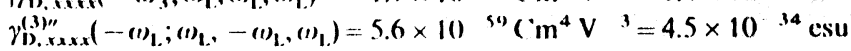


lincar susceptibility, $\chi^{(3)}=\chi_{x x x x x}^{(3)}\left(-(1)_{3} ;(1)_{1},(1)_{1},()_{1}\right)$, was derived in [7 and 17]. The result is

$\eta=\frac{4 \pi^{2} \tilde{i}_{3}^{2}\left\{\exp \left(-3 \alpha_{1} l\right)+\exp \left(-\alpha_{3} l\right)-2 \exp \left[-\left(\alpha_{3}+3 \alpha_{L}\right) l / 2\right] \cos (\Delta k l)\right\}}{3^{3 / 2} n_{3} n_{L}^{3} s_{0}^{2} c_{0}^{2}\left[\left(\alpha_{3}-3 \alpha_{1}\right)^{2} / 4+\Delta k^{2}\right]}\left|\chi^{(3)}\right|^{2} I_{\text {OL }}^{2}$.

Equation (2) applies to a temporal and spatial Gaussian input pulse shape

$\left[I_{1}\left(t^{\prime}, r\right)=I_{\mathrm{OL}} \exp \left(-t^{\prime 2} / t_{0}^{2}-r^{2} / r_{0}^{2}\right), t^{\prime}=t-n_{\mathrm{L}} z / c_{0}\right]$.

$\tilde{v}_{3}=v_{3} / c_{0}$ is the wavenumber of the third harmonic light. $c_{0}$ is the vacuum light velocity. $\varepsilon_{0}$ is the dielectric permittivity. $\alpha_{1}$ and $\alpha_{3}$ are the absorption coefficients at $v_{\mathrm{L}}$ and $v_{3}$, respectively. $n_{\mathrm{L}}$ and $n_{3}$ are the corresponding refractive indices. The wavevector mismatch, $1 k=k_{3}-3 k_{1}=6 \pi \tilde{v}_{\mathrm{L}}\left(n_{3}-n_{\mathrm{L}}\right)$, is zero in case of phasematching. The absorption coefficients and refractive indices are listed in Table 1.

Fitting (2) to the experimental points of Fig. 4 in the intensity region $I_{\mathrm{OL}}<10^{10} \mathrm{~W} / \mathrm{cm}^{2}$ gives a value of $\left|\chi^{(3)}\right|$ $=(2 \pm 0.1) \times 10^{-22} \mathrm{~m}^{2} \mathrm{~V}^{-2}$ at the phase-matching concentration of $C_{\mathrm{PM}}=0.0825 \mathrm{~mol} / \mathrm{dm}^{3}$.

The third-order nonlinear susceptibility of the solvent hexafluoroisopropanol was measured separately in a special cell assembly as described in [17]. The obtained nonlinear susceptibility of the solvent is

$$
\begin{aligned}
\chi_{S}^{(3)} & =\chi_{S, x x x x}^{(3)}\left(-\omega_{3} ; \omega_{L}, \omega_{L}, \omega_{L}\right) \\
& =(1.4 \pm 0.2) \times 10^{-23} \mathrm{~m}^{2} \mathrm{~V}^{-2} .
\end{aligned}
$$

At the phase-matching concentration $\chi_{\mathrm{s}}^{(3)}$ is negligibly small compared to $\left|\chi^{(3)}\right|$ and the measured nonlinear susceptibility $\left|\chi^{(3)}\right|$ is set equal to the nonlinear dye susceptibility $\left|\chi_{\mathrm{D}}^{(3)}\right|$. (The real and imaginary part of $\chi_{\mathrm{D}}^{(3)}$ are not separately determined in the present work, see [7]).

The relation between the dye susceptibility, $\chi_{\mathrm{D}}^{(3)}$, and the dye hyperpolarizability, $\gamma_{b}^{(3)}$, is [7]

$$
\begin{aligned}
& \chi_{\mathrm{D} . \times x \times x}^{(3)}\left(-\omega_{3} ;\left(\omega_{\mathrm{L}}, \omega_{\mathrm{L}}, \omega_{\mathrm{L}}\right)=\frac{N_{\mathrm{D}} L\left(-\omega_{3} ; \omega_{\mathrm{L}}, \omega_{\mathrm{L}}, \omega_{\mathrm{L}}\right)}{\varepsilon_{0}}\right. \\
& \quad \times \gamma_{\mathrm{D},{ }_{x \times x \times x}^{(3)}\left(-\omega_{3} ; \omega_{\mathrm{L}}, \omega_{\mathrm{L}}, \omega_{\mathrm{L}}\right)}
\end{aligned}
$$

$N_{\mathrm{D}}=C N_{\mathrm{A}}$ is the number density of dye molecules. $C$ is the dye concentration, and $N_{A}=6.022045$ $\times 10^{23} \mathrm{~mol}^{-1}$ is the Avogadro number. $11-\left(\omega_{3} ;()_{1}, \omega_{1},\left(\omega_{1}\right)=\left(n_{3}^{2}+2\right)\left(n_{\mathrm{L}}^{2}+2\right)^{3} / 81\right.$ is the Lorentz local field correction factor. The obtained hyperpolarizability of PYC is $\left|\gamma_{0}^{(3)}\right|=(1.7 \pm 0.2)$ $\times 10^{59} \mathrm{Cm}^{4} / \mathrm{V}^{3}$. Similar, the hyperpolarizability of the solvent hexafluoroisopropanol is determined to be $r_{\mathrm{S}}^{(3)}=(1 \pm 0.1) \times 10^{-62} \mathrm{Cm}^{4} \mathrm{~V}^{-3} \quad\left(N_{\mathrm{S}}=\varrho N_{\mathrm{A}} / m_{\mathrm{M}}=5.8\right.$ $\times 10^{21} \mathrm{~cm}^{-3}$, density $\varrho=1.62 \mathrm{~g} / \mathrm{cm}^{3}$, molecular mass $\left.m_{M}=168.04 \mathrm{~g} / \mathrm{mol}\right)$.

The two-photon absorption of laser light in dye solutions is determined by

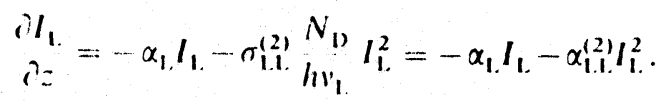

The first term gives the linear absorption. It is due to solvent absorption. $\sigma_{\mathrm{l} . \mathrm{l}}^{(2)}$ is the two-photon absorption cross-section of the dye molecules for the simultaneous absorption of two photons of frequency $v_{1}$. The twophoton absorption coefficient, $\alpha_{\mathrm{LL}}^{(2)}$, is related to the two-photon absorption cross-section, $\sigma_{\mathrm{LL}}^{(2)}$, by $\alpha_{\mathrm{l}, \mathrm{l}}^{(2)}$. $=\sigma_{\mathrm{LL}}^{(2)} N_{\mathrm{D}} / h v_{\mathrm{L}}$. Excited state absorption at frequency $v_{\mathrm{L}}$. from the populated $S_{1}$-state to higher lying singlet states is not included. The inclusion would require a more rigorous treatment $[14,18,19]$. The intensity transmission, $T_{I}=I_{\mathrm{L}}(l) / I_{\mathrm{L}}(0)$, is obtained by integrating of Eq. 4. The result is [20]

$$
T_{l}\left(r, t^{\prime}\right)=\frac{\exp \left(-\alpha_{\mathrm{L}} l\right)}{1+I_{\mathrm{L}}(r, t) \alpha_{\mathrm{LL}}^{(2)}\left[1-\exp \left(-\alpha_{\mathrm{L}} l\right)\right] / \alpha_{\mathrm{L}}}
$$

The time integrated intensity transmission is given by

$T_{t}(r)=\frac{\int_{-\infty}^{\infty} T_{l}\left(r, t^{\prime}\right) I_{\mathrm{L}}\left(r, t^{\prime}\right) d t^{\prime}}{\int_{-\infty}^{\infty} I_{\mathrm{L}}\left(r, t^{\prime}\right) d t^{\prime}}$.

The energy transmission, $T_{\mathrm{E}}=W_{\mathrm{L}}(l) / W_{\mathrm{L}}$ ( $W_{\mathrm{L}}$ input pulse energy), is found by integration over the temporal and spatial pulse distribution

$T_{\mathrm{E}}=\frac{\int_{0}^{\infty} r\left[\int_{-\infty}^{\infty} T_{I} I_{\mathrm{L}}\left(r, t^{\prime}\right) d t^{\prime}\right] d r}{\int_{0}^{\infty} r\left[\int_{-\infty}^{\infty} I_{\mathrm{L}}\left(r, t^{\prime}\right) d t^{\prime}\right] d r}$

Equations (4-7) are valid as long as ground-state depopulation by two-photon absorption is negligibly small. Otherwise $N_{\mathrm{D}}$ has to be replaced by $N_{1}=N_{\mathrm{D}}-N_{2}$ (Fig. 6) and a coupled differential equation system has to be solved (4 and 14 below) [14].

$\sigma_{\mathrm{LL}}^{(2)}$ is determined by fitting Eq. 7 to the experimental energy transmission points of the 0.1 molar dye solution in a $1 \mathrm{~cm}$ long cell (circles and curve 3 of Fig. 5). The obtained two-photon absorption crosssection is $\sigma_{\mathrm{LI}}^{(2)}=(1.8 \pm 0.2) \times 10^{-49} \mathrm{~cm}^{4} \mathrm{~s}$. The good fit of the calculated curves to the experimental points indicates negligible $S_{1}-S_{n}$ excited-state absorption at the laser frequency $v_{\mathrm{L}}$.

The two-photon absorption, as the third harmonic generation, is a third-order nonlinear optical process and may be expressed by a third-order nonlinear susceptibility or hyperpolarizability. $\alpha_{\mathrm{LL}}^{(2)}$ is related to the imaginary part, $\chi_{D}^{(3) \prime \prime}$, of the third-order susceptibility $\chi_{1}^{(3), x \times x x}\left(-()_{1} ; \omega_{1}, \cdots-()_{1}, \omega_{1}\right)$ by $[21]$

$\chi_{i}^{(3), \ldots \ldots \times x}\left(-(1)_{1},(1)_{1},-(1)_{1},(1)_{1}\right)=\frac{c_{0}^{2} n_{1}^{2} x_{0}}{6(1)_{1}} \alpha_{1.1}^{(2)}$ 
(11) $=2 \pi v_{1}$ is the angular frequency of the fundamental laser. The imaginary part of the two-photon absorption hyperpolarizability is related to the two-photon absorption cross-section by

$$
\begin{aligned}
& \gamma^{\prime(3),{ }_{x \times x x}}\left(-\omega_{L} ; \omega_{L},-\omega_{L}, \omega_{L}\right) \\
& =\frac{\varepsilon_{0}}{\left.N_{D} L-\omega_{L} ; \omega_{L},-\omega_{L}, \omega_{L}\right)}
\end{aligned}
$$

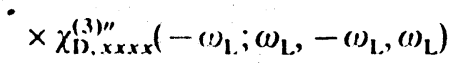

$$
\begin{aligned}
& =\frac{n_{\mathrm{L}}^{2} c_{0}^{2} \varepsilon_{0}^{2}}{6 \omega_{\mathrm{L}} N_{\mathrm{D}} L\left(-\omega_{\mathrm{L}} ; \omega_{\mathrm{L}},-\omega_{\mathrm{L}}, \omega_{\mathrm{L}}\right)} \alpha_{\mathrm{LL}}^{(2)} \\
& =\frac{\pi n_{\mathrm{L}}^{2} c_{0}^{2} \varepsilon_{0}^{2}}{3()_{\mathrm{L}}^{2} h L\left(-\omega_{\mathrm{L}} ; \omega_{\mathrm{L}},-\omega_{\mathrm{L}}, \omega_{\mathrm{L}}\right)} \sigma_{\mathrm{LL}}^{(2)},
\end{aligned}
$$

$L\left(-\omega_{\mathrm{L}} ; \omega_{\mathrm{L}},-\omega_{\mathrm{L}}, \omega_{\mathrm{L}}\right)=\left(n_{\mathrm{L}}^{2}+2\right)^{4} / 81$ is the Lorentz local field correction factor. The obtained $\chi_{D}^{(3) "}$ and $\gamma_{D}^{(3) "}$ values are listed in Table 1 . The two-photon hyperpolarizability, $\gamma_{D, x x x x}^{(3) \prime \prime}\left(-\omega_{L} ; \omega_{L},-\omega_{L}, \omega_{L}\right)$, is a factor of 3.2 larger than the third-harmonic hyperpolarizability, $\left|\gamma_{\text {b.xxx.x }}^{(3)_{\text {x }}}\left(-()_{3} ; \omega_{\mathrm{L}}, \omega_{\mathrm{L}}, \omega_{\mathrm{L}}\right)\right|$.

The two-photon absorption of pump laser light reduces slightly the third harmonic generation, because of reduced pump pulse intensity

$\left[I_{3}\left(r, t^{\prime}, l\right) \propto I_{i}^{3}\left(r, t^{\prime}, l\right)\right]$.

The responsible pump pulse intensity is approximated by the output pulse intensity since the short interaction length, $l_{1 \text {. THG }}\left(l_{1, \text { THG }} \simeq 3 \alpha_{3}^{-1}\right)$ limits the main third harmonic contribution to the exit region of the cell. Equation (2) modifies to

$\eta^{\prime} \simeq T_{\mathrm{i}}^{3} \eta$.

The solid curve 2 of Fig. 4 represents $\eta^{\prime}$ for the $1 \mathrm{~mm}$ cell. Curve 1 gives the uncorrected energy conversion $\eta$ of Eq. 2 for the same sample.

Besides the two-photon absorption of laser light the simultaneous absorption of third harmonic light and pump laser light occurs. This two-photon absorption process is caused by [22]

$$
\begin{aligned}
\alpha_{\mathrm{L}, 3}^{(2)} & =\stackrel{N_{\mathrm{D}}}{h v_{\mathrm{L}}} \sigma_{\mathrm{L},(2)}^{(2)} \\
& =\frac{12(1)_{3}}{c_{0}^{2} n_{1} n_{3}} \chi_{x \times x \times x}^{(3) \prime \prime}\left(-\omega_{3} ; \omega_{\mathrm{L}},-\omega_{\mathrm{L}}, \omega_{3}\right),
\end{aligned}
$$

The responsible differential equation is [23]

$$
\frac{\partial_{3}}{\partial_{2}}=-\alpha_{1.3}^{(2)} I_{1} I_{3}
$$

and the third harmonic conversion efficiency reduces approximately to

$\eta^{\prime \prime} \simeq \eta^{\prime} \exp \left(-\alpha_{1.3}^{(2)} I_{1} l_{l, \text { THG }}\right)$.
Curve 3 in Fig. 4 is calculated by use of Eq. 13 assuming $\sigma_{\mathrm{L}, 3}^{(2)}=6 \sigma_{\mathrm{LL}}^{(2)}$ [assumption of equal nonlinear susceptibility, i.e. $\chi_{x x x x}^{(3) \prime \prime}\left(-()_{3} ; \omega_{\mathrm{L}},-\omega_{\mathrm{L}}, \omega_{3}\right)=\chi_{x x x x}^{(3) \prime \prime}\left(-\omega_{1} ; \omega_{\mathrm{L}}\right.$, $\left.-\omega_{\mathrm{L}}, \omega_{\mathrm{L}}\right)$, see Eqs. 8 and 11$]$ and $T_{\mathrm{L}}=I_{\mathrm{OL}}(l) / 2=I_{\mathrm{OL}} T_{l} / 2$ (factor $1 / 2$ takes care of temporal and spatial intensity distribution). The reduction of the energy conversion by two-photon absorption of third harmonic light is small because of the short interaction length $l_{\text {I.THC; }}$ (large linear absorption coefficient $\alpha_{3}$ ).

The deviation of the experimental points at high pump pulse intensity from $\eta^{\prime \prime}$ is thought to be due to $S_{1}-S_{n}$ excited state absorption of the generated third harmonic light. In the following (14-18) analytical estimates of the $S_{1}$-state level population, $N_{2}$, the excited state absorption (cross-section $\sigma_{3, \text { ex }}$ ), and the amplified spontaneous emission are given. A detailed analysis requires numerical simulations [22].

The $S_{1}$ state is populated by two-photon absorption of laser light according to [14]

$\frac{\partial N_{2}}{\partial t}=\frac{\sigma_{\mathrm{LL}}^{(2)}\left(N_{\mathrm{D}}-N_{2}\right)}{2\left(h v_{\mathrm{L}}\right)^{2}} I_{\mathrm{L}}^{2}$.

Equation (14) is valid only for times $t$ short compared to the fluorescence lifetime of level $2\left(\tau_{\mathrm{F}} \approx 10 \mathrm{ps}\right.$ for PYC at $C_{\mathrm{PM}}=0.0825 \mathrm{~mol} / \mathrm{dm}^{3}$ [14]) and if the $S_{1}$ state is not depopulated markedly by amplified spontaneous emission within time $t$. Formal integration of (14) gives for $t \gtrsim t_{0}\left(t_{0}\right.$ is half the $1 / e$ pump pulse width)

$N_{2}(l) \simeq \frac{\sigma_{\mathrm{LL}}^{(2)}\left[N_{\mathrm{D}}-N_{2}(l)\right] \bar{I}_{\mathrm{L}}^{2} t_{\mathrm{eff}}}{2\left(h v_{\mathrm{L}}\right)^{2}}$

$t_{\text {eff }}$ is given approximately by the pulse duration $\Delta t_{\mathrm{L}}$ (FWHM) of the pump pulse $\left(\Delta t_{\mathrm{L}} \simeq 5\right.$ ps in our experiments). $\bar{I}_{\mathrm{L}}$ is given approximately by $\bar{I}_{\mathrm{L}} \simeq I_{\mathrm{OL}} T_{\mathrm{l}} / 2$. Solving (15) to $N_{2}$ and using $t_{\text {eff }}=\Delta t_{\mathrm{L}}$ gives

$\mathrm{N}_{2}(l) \simeq N_{\mathrm{D}}\left[1+\frac{8\left(h v_{\mathrm{L}}\right)^{2}}{\sigma_{\mathrm{LL}}^{(2)} I_{\mathrm{OL}}^{2} T_{1}^{2} \Delta t_{\mathrm{L}}}\right]^{-1}$.

$N_{2}(l)$ versus $I_{\mathrm{OL}}$ is plotted in Fig. 7 for $l=1 \mathrm{~mm}$ (curve 1) and $0.1 \mathrm{~mm}$ (curve 2).

In order to take the excited-state absorption of third harmonic light into account, $\alpha_{3}$ of (2) has to be replaced by

$$
\begin{aligned}
\alpha_{3}^{\prime}(l) & =\alpha_{3}(l)+\alpha_{3, \mathrm{ex}}(l) \\
& =\sigma_{3}\left[N_{\mathrm{D}}-N_{2}(l)\right]+N_{2}(l) \sigma_{3, \mathrm{ex}} .
\end{aligned}
$$

In Fig. 4, curve 4 is fitted to the experimental points belonging to the $1 \mathrm{~mm}$ cell length in the intensity region $I_{\mathrm{OL}}<8 \times 10^{10} \mathrm{~W} / \mathrm{cm}^{2}$. The obtained best-fitting excited-state absorption cross-section is $\sigma_{3, \mathrm{ex}}=2.6$ $\times 10^{-16} \mathrm{~cm}^{2}$. Using this $\sigma_{3, \text { ex }}$ value and $N_{2}(l)$ of (16) the curves 4,5 , and 6 of Fig. 4 are calculated for the sample lengths $l=1 \mathrm{~mm}, 0.2 \mathrm{~mm}$, and $0.1 \mathrm{~mm}$, respec- 


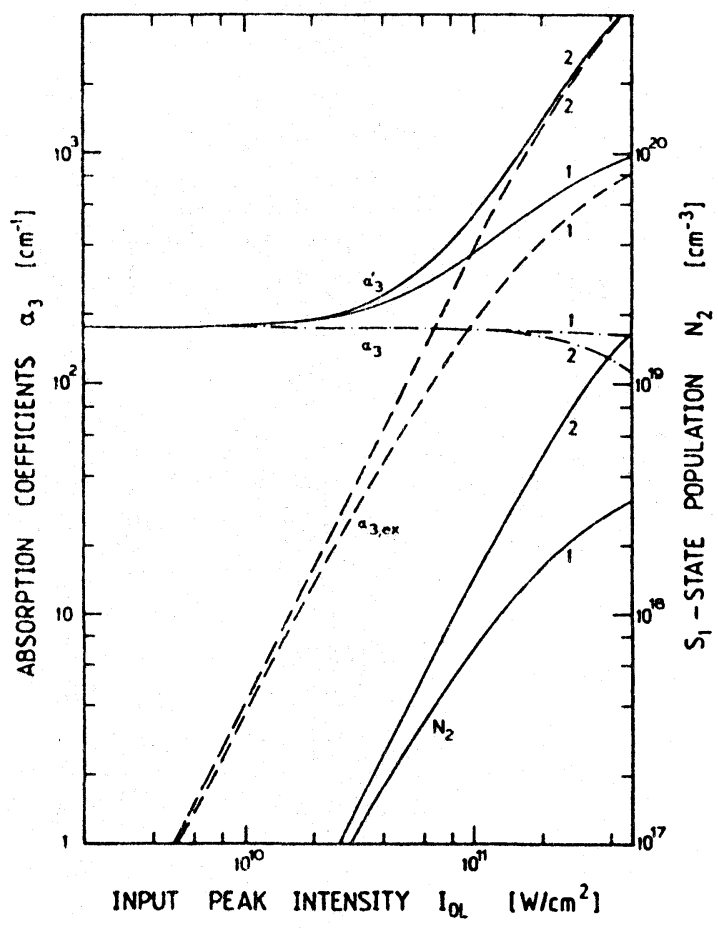

Fig. 7. $S_{1}$-state population, $N_{2}(l)$, and absorption coefficients, $\alpha_{3}^{\prime}(l), \alpha_{3}(l)$, and $\alpha_{3, \text { cx }}(l)$, versus input pulse peak intensity for $l=1 \mathrm{~mm}$ (curves 1 ) and $l=0.1 \mathrm{~mm}$ (curves 2). Dye concentration $C=C_{\mathrm{PM}}=0.0825 \mathrm{~mol} / \mathrm{dm}^{3}$

tively. The same parameters are used for the calculation of the concentration dependent normalized energy transmission curves of Fig. $3(l=1 \mathrm{~mm}$, curve 1 : $I_{\mathrm{OL}}=4 \times 10^{9} \mathrm{~W} / \mathrm{cm}^{2}$ and curve $2: I_{\mathrm{OL}}=10^{11} \mathrm{~W} / \mathrm{cm}^{2}$ ). The deviation of the experimental circles from curve 1 seems to be due to deviations of the refractive indices from the assumed linear concentration behaviour [24] $\left\{\chi^{(1)}(\omega)=n^{2}(\omega)-1=\chi_{s}^{(1)}(\omega)+N_{\mathrm{D}} L(\omega) \gamma^{(1)} / \varepsilon_{0}\right.$ with $L(\omega)$ $=\left[n^{2}(\omega)+2\right] / 3$ and $\left.\chi_{s}^{(1)}(\omega)=n_{s}^{2}(\omega)-1\right\}$. The broadening of the peak of curve 2 is caused by the enlarged absorption coefficient $\alpha_{3}^{\prime}(l)$.

The dependence of the absorption cross-sections $\alpha_{3}^{\prime}(l), \alpha_{3}(l)$, and $\alpha_{3, \text { ex }}(l)$ on the input peak intensity are depicted in Fig. 7 for $l=1 \mathrm{~mm}$ (curves 1 ) and $l=0.1 \mathrm{~mm}$ (curves 2). The pump pulse intensity, $I_{\mathrm{OL}}$, where $\alpha_{3}^{\prime}(l)$ becomes equal to $2 \alpha_{3}(0)=2 \sigma_{3} N_{\mathrm{D}}$ is called saturation intensity, $I_{\mathrm{L} . \mathrm{S}}^{\mathrm{THG}}$, and the corresponding level population, $N_{2}(l)$, is called saturation population $N_{2 . s}^{\text {THG }}$. Above $I_{\mathrm{L} . \mathrm{s}}^{\text {rifg }}$ strong saturation in third harmonic generation occurs $\left[I_{L . S}^{\mathrm{THG}}=1 \times 10^{11}, 7.5 \times 10^{10}\right.$, and $7 \times 10^{10} \mathrm{~W} / \mathrm{cm}^{2}$, for $l=1 \mathrm{~mm}, 0.2 \mathrm{~mm}$, and $0.1 \mathrm{~mm}$, respectively]. A decrease of $\alpha_{3}$ by a factor of 40 (minimum absorption of PYC at $375 \mathrm{~nm}$ ) would lower $I_{1 . . \mathrm{S}}^{111(\mathrm{~S})} 101.4 \times 10^{10} \mathrm{~W} / \mathrm{cm}^{2}$, the interaction length would increase by a factor of $40,\left(l_{\mathrm{I} \text { THG }}=0.68 \mathrm{~cm}\right)$, and the third harmonic conversion efficiency would rise to ap- proximately $3 \times 10^{-3}$ at $I_{\mathrm{OI}}=I_{\mathrm{I} . \mathrm{S}}^{\mathrm{THG}}$ and $l=l_{\mathrm{I} \text {. THG }}$ (other parameters are the same as for calculations of curves 4 , 5 , and 6 in Fig. 4). This estimate indicates the importance of weak absorption at $v_{3}$ for efficient third harmonic generation.

The deviations of the experimental points from the calculated curves 4,5 , and 6 of Fig. 4 above $10^{11} \mathrm{~W} / \mathrm{cm}^{2}$ are thought to be due to the occurrance of amplified spontaneous emission which reduces the level population $\mathrm{N}_{2}$ and therefore increases the third harmonic conversion efficiency (lowering of $\alpha_{3, \mathrm{ex}}$ ). The occurrance of amplified spontaneous emission was verified experimentally [e.g., for $I_{\mathrm{OL}} \simeq 10^{11} \mathrm{~W} / \mathrm{cm}^{2}$, $l=1 \mathrm{~mm}, C=C_{\mathrm{PM}}=0.0825 \mathrm{~mol} / \mathrm{dm}^{3}$ strong amplified spontaneous emission $\left(W_{\mathrm{ASE}} / W_{\mathrm{L}} \simeq 0.01\right)$ within a small divergence angle in forward direction at $\lambda_{\mathrm{ASF}} \simeq 600 \mathrm{~nm}$ with $\Delta \lambda_{\text {ASE }} \simeq 10 \mathrm{~nm}$ is observed]. Depopulation of the $S_{1}$-state by amplified spontaneous emission becomes relevant for gain factors $G \gtrsim \exp (20)$ [14], where

$$
\begin{aligned}
G \simeq & \exp \left\{\left[\sigma_{\mathrm{em}}^{\mathrm{ASE}}\left(N_{2}-N_{3}\right)-\sigma_{\mathrm{ex}}^{\mathrm{ASE}} N_{2}\right.\right. \\
& \left.\left.-\sigma_{\mathrm{A}}\left(v_{\mathrm{ASE}}\right)\left(N_{\mathrm{D}}-N_{2}\right)\right] l\right\} .
\end{aligned}
$$

$\sigma_{\mathrm{A}}\left(v_{\mathrm{ASE}}\right)$ is the apparent ground state absorption crosssection at frequency $v_{\text {ASE. }}$.

In case of fast depopulation of the terminal level of amplified spontaneous emission (level 3 in Fig. 6, $\tau_{v} \rightarrow O$ ) the $S_{1}$-state level population becomes limited to

$N_{2, \max }=\frac{20+N_{\mathrm{D}} \sigma_{\mathrm{A}}\left(v_{\mathrm{ASE}}\right) l}{\left[\sigma_{\mathrm{em}}^{\mathrm{ASE}}-\sigma_{\mathrm{ex}}^{\mathrm{ASE}}+\sigma_{\mathrm{A}}\left(v_{\mathrm{ASE}}\right)\right] l}$.

For $l=1 \mathrm{~mm} \quad$ it is $\lambda_{\mathrm{ASE}}=600 \mathrm{~mm}, \quad \sigma_{\mathrm{em}}^{\mathrm{ASE}}=1.3$ $\times 10^{-16} \mathrm{~cm}^{2}$ and $\sigma_{\mathrm{A}}\left(v_{\mathrm{ASE}}\right)=1.5 \times 10^{-19} \mathrm{~cm}^{2}$. Assuming $\sigma_{\mathrm{ex}}^{\mathrm{ASE}}=1 \times 10^{-17} \mathrm{~cm}^{2}$, the population limit becomes $N_{2, \max }=1.7 \times 10^{18} \mathrm{~cm}^{-3}$. If the lower level of the amplified spontaneous emission transition has a finite lifetime ( $\tau_{v}>0$, typical value $\tau_{v} \simeq 4$ ps [25]) then only $N_{2}-N_{3}$ approaches a limiting value and $N_{2}$ continues to rise with pump pulse intensity (bottle neck effect).

The described reduction in the growth of $N_{2}$ is thought to be responsible for the approximately constant energy conversion of third harmonic light at intensities above $10^{11} \mathrm{~W} / \mathrm{cm}^{2}$ (experimental points of Fig. 4 compared to decreasing curves 4,5 , and 6). A theoretical fitting to the experimental points requires a numerical analysis of third harmonic generation in presence of two-photon absorption, excited-statc absorption, and amplified spontaneous emission [22].

\section{Conclusions}

The phase-matched third harmonic generation in the dye PYC dissolved in hexafluoroisopropanol allows an energy conversion of about $2 \times 10^{-4}$ for 
$\mathrm{Nd}$ : glass laser picosecond pump pulses of intensities $I_{01} \geqq 10^{11} \mathrm{~W} / \mathrm{cm}^{2}$. The conversion efficiency is limited at high pump pulse intensities by two-photon absorption and excited-state absorption of third harmonic light. The amplified spontaneous emission reduces the $S_{1}$-state population within the pump pulse duration and acts against the decrease of the conversion efficiency by excited state absorption. Third harmonic light generation with a conversion efficiency up to the per cent region should be possible in dye solutions with lower ground-state absorption cross-sections $\sigma_{3}$. Lower excited-state absorption cross-sections, $\sigma_{3, \text { ex }}$, higher effective stimulated emission cross-sections, $\sigma_{\mathrm{em}}^{\mathrm{ASE}}-\sigma_{\mathrm{ex}}^{\mathrm{ASE}}$, and fast $S_{0}$-state thermalisation times $\tau_{n} \rightarrow 0$ would increase the conversion efficiency at high pump pulse intensities. Dyes with $S_{1}$-state lifetimes less than the pump pulse duration $\left(\tau_{\mathrm{F}}<\Delta t_{\mathrm{L}}\right)$ would also reduce the accumulation of population in the $S_{1}$ state and increase the conversion efficiency at high pump pulse intensities.

The third harmonic light generation was also studied in the dyes $1,3,3,1^{\prime}, 3^{\prime}, 3^{\prime}$-hexamethylindocarbocyanine iodide (HMICI) [5] and safranine $T$ which have, similar to PYC, low absorption cross-sections at $\lambda_{3}=351.3 \mathrm{~nm}$ in the solvent hexafluoroisopropanol (HMICI: $\sigma_{3} \simeq 2.6 \times 10^{-18} \mathrm{~cm}^{2}$, safranine $T: \sigma_{3} \simeq 2.3 \times 10^{-18} \mathrm{~cm}^{2}$ ). The phase-matching concentrations of these dyes are $C_{\mathrm{PM}}=0.08 \mathrm{~mol} / \mathrm{dm}^{3}$ (HMICl) and $C_{\mathrm{PM}}=0.33 \mathrm{~mol} / \mathrm{dm}^{3}$ (safranine $T$ ). The obtained maximum conversion efficiencies are similar to PYC (HMICI: $\eta \simeq 4 \times 10^{-4}$ at $I_{\mathrm{OL}} \simeq 3 \times 10^{11} \mathrm{~W} / \mathrm{cm}^{2}$ and $l=0.1 \mathrm{~mm}$; safranine $T: \eta \simeq 8 \times 10^{-5}$ at $I_{\mathrm{OL}} \simeq 3$ $\times 10^{11} \mathrm{~W} / \mathrm{cm}^{2}$ and $\left.l=0.1 \mathrm{~mm}\right)$.

Acknowledgements. The authors are very indebted to Dr. U. Mayer of BASF Ludwigshafen for providing the dye PYC and for helpful discussions. They are grateful to Dr. H. J. Sasse (BASF) for valuable information. They thank the Deutsche Forschungsgemeinschaft and the Fonds der chemischen Industrie for financial support and the Rechenzentrum of the University of Regensburg for disposal of computer time.

\section{References}

1. P.P. Bey, I.E. Giuliani, H. Rabin: Phys. Rev. Lett. 19, 819 (1967)

2. P.P. Bey, I.E. Giuliani, H. Rabin: IFEE J. QE-4, 932 (1968)

3. R.K. Chang, L.K. Galbraith: Phys. Rev. 171, 993 (1968)

4. P.P. Bey, J.F. Giuliani, H. Rabin: IEEE J. QE-7, 86 (1971)

5. J.C. Dicls, F.P. Schäfer: Appl. Phys. 5, 197 (1974).

6. L.I. Alperovich, T.B. Bavaev, V.V. Shabalov: Sov. J. Appl. Spectrosc. 26, 196 (1977)

7. W. Leupacher, A. Penzkofer: Appl. Phys. B 36, 25 (1985)

8. A. Penzkofer, D. von der Linde, A. Laubereau: Opt. Commun. 4, 377 (1972)

9. Y. Lu, A. Penzkofer: Appl. Opt. 25, 221 (1986)

10. A. Penzkofer, W. Leupacher, B. Meier, B. Runde, K.H. Drexhage: Chem. Phys. 115, 143 (1987)

11. W. Falkenstein, A. Penzkofer, W. Kaiser: Opt. Commun. 27, 151 (1978)

12. A. Penzkofer, W. Falkenstein: Opt. Quantum Electron. 10, 399 (1978)

13. A. Penzkofer: Appl. Phys. B 40, 85 (1986)

14. P. Sperber, A. Penzkofer: Opt. Quantum Electron. 18 (1986) (to be published)

15. J.F. Reintjes: Nonlinear Parametric Processes in Liquids and Gases (Academic, Orlando 1984) p. 240

16. J.F. Reintjes: In Laser Handbook, Vol. 5, ed. by M. Bass and M.L. Stitch (North-Holland, Amsterdam 1985) Chap. 1

17. M. Thalhammer, A. Penzkofer: Appl. Phys. B 32, 137 (1983)

18. A. Penzkofer, W. Leupacher: Opt. Quantum Electron. 19 (1987)

19. A. Penzkofer, W. Falkenstein: Opt. Commun. 16, 247 (1976)

20. A. Penzkofer, W. Kaiser: Appl. Phys. Lett. 21, 427 (1972)

21. P. Sperber, A. Penzkofer: Opt. Quantum Electron. 18, 145 (1986)

22. A. Penzkofer, W. Leupacher: To be published

23. A. Penzkofer, W. Falkenstein, W. Kaiser: Appl. Phys. Lett. 28, 319 (1976)

24. H.J. Lehmeier, W. Leupacher, A. Penzkofer: Opt. Commun. 56, 67 (1985)

25. D. Ricard, J. Ducuing: J. Chem. Phys. 62, 3616 (1975)

26. R.W. Minck, R.W. Terhune, C.C. Wang: Appl. Opt. 5, 1595 (1966)

27. Technical report DP-4A of Dupont on hexafluoroisopropanol (1968) 